\title{
Comparison of different methods of fixation in Papanicolaou staining of cervical smears: Wet-fixation and rehydration of air dried smears
}

\author{
Narayanan O. N. ${ }^{1}$, Bai A. ${ }^{2}$ \\ ${ }^{1}$ Dr. Navya Narayanan O., Associate Professor, Department of Pathology, ${ }^{2}$ Dr. Ambika Bai, Professor and HOD, \\ Department of Obstetrics \& Genecology, both authors are affiliated with Sree Narayana Institute of Medical Sciences, \\ Ernakulum, Kerala, India.
}

Corresponding Author: Dr. Navya Narayanan O, Associate Professor, Department of Pathology, Sree Narayana Institute of Medical Sciences, Ernakulum, Kerala, India. E-mail: navyanarayano@gmail.com

\begin{abstract}
Introduction: Cervical cancer is a major cause of death in women in all over the world. Cytological screening by Papanicolaou (Pap) smear had reduced significantly the occurrence of invasive cervical cancer. Obtaining a properly stained smear is needed for correct diagnosis which is often not possible due to improper fixation and drying artefacts. Objective: Our study tried to compare the morphology of the cervical smears fixed by two different fixation techniques, rehydration of air-dried smears (AD) versus wet fixation (WF). Design: This is a cross sectional study conducted in the department of pathology, Sree Narayana institute of medical sciences, Ernakulum, Kerala. Subjects: We collected cervical smears from three hundred patients in 24-72 years who attended our gynaecology department. Methods: Two slides are collected per patient. One of slides is immediately wet fixed in 95\% ethanol (WF). The other slide was air dried and rehydrated for prior to routine staining by using glycerol saline rehydration method. Then the slides were stained by standard Papanicolaou method and paired slides were compared. Result: Most of the patients (51\%) included in the study belonged to 35-45- years of age years, presented with complaints of discharge per vaginum. Air dried smears showed better cytoplasmic staining, distinct nuclear borders, clear background and low rate of cytolysis compared to wet fixed smears. Conclusions: Because of many advantages compared to the conventional wet fixation, air dried smears can be used routinely or as an alternative in mass cervical cancer screening programmes
\end{abstract}

Key words: Cervical smear, Wet fixation, Rehydration

\section{Introduction}

Cervical cancer contributes to the large majority of malignancy affecting females in India [1]. It is a major cause of death in women. Since the development of invasive carcinoma take many years to precede from the precursor intraepithelial lesions caused by HPV DNA 16 and 18. This longer duration made it able to diagnose cervical lesions at an early stage by a cytological screening by Papanicolaou smear [2]. Routine screening programmes by the above method had reduced significantly the occurrence of invasive cervical cancer in the developed countries.

The pap test is a simple outpatient test done by using spatula or Endo-cervical Brush, Cytobrush or Cervex. After smear collection, the obtained sample is evenly smeared on to centre of the non-frosted area of the glass slide and it is fixed immediately in $95 \%$ ethyl alcohol

Manuscript received: $28^{\text {th }}$ December 2018

Reviewed: $5^{\text {th }}$ January 2019

Author Corrected: $10^{\text {th }}$ January 2019

Accepted for Publication: 14 $4^{\text {th }}$ January 2019 for minimum of 15 minutes and stained with Papanicolaou stain which will highlight cellular morphology, nuclear chromatin and other cells in the background [3]. But, unfortunately, it is often seen that improper fixation and drying artefacts can occur either due to inadequate training of workers, heavy workload or from short supply and storage of alcohol which is very essential for fixation. That will cause repeating of smears and increasing workload or missing the patients [4].That difficult situation demanded another alternative method to wet fixation. One possible method is airdrying of cervical smears and rehydration of these smears just prior to alcohol fixation [5].

The current study is enthusiastically undertaken to evaluate the possibility of routine use of rehydration of air-dried Pap smears in a tertiary care hospital in Kerala, south India. Rehydration can be obtained by immersing cytological specimens in 50\% aqueous solution of glycerin for three minutes or in normal 
saline for 30 seconds. These air dried smears can also be later used for immunostaining, in addition to pap staining. This method has been offered for fine needle aspiration (FNA), effusion cytology and exfoliated cells, but few reports regarding its use as a substitute technique for cervical smears are available [6].

\section{Aims and Objectives}

The objective of the study is to compare the morphology of air dried glycerol rehydrated pap smears with traditional wet alcohol fixed pap smears in the screening of cervical lesions in a tertiary care centre in Kerala

1. To compare the morphology of the cervical smears fixed by two different fixation techniques, rehydration of air-dried smears (AD) versus wet fixation (WF).

2. To find out whether rehydration techniques can be adopted as an alternative method, along with routine smears, especially in high-volume, resource-limited settings.

\section{Material \& Methods}

This is a cross sectional study conducted in the department of pathology Sree Narayana Institute of Medical Sciences, Ernakulum, Kerala. We collected three hundred cervical smears over a period of one year from patients who attended our gynaecology department. The age of the women from whom specimens collected ranged from 24-72 years. Menstruating and pregnant women were excluded from the study.

Study setting: Department of pathology, Sree Narayana institute of medical sciences

\section{Original Research Article}

Study duration: one year

Sample type: Cervical smears

Sample size: 300 smears

Sampling method: Convenience sampling

Ethical issue: informed consent taken before procedure

Data storage and Analysis

Method- This study is done in co-operation with OBG department in our medical college. We planned to collect 300 pap smears. Before sample collection basic data including name, age, gender, marital status, menstrual history, obstetric history, and detailed medical or surgical history was collected from the patient. The cervical smears were taken in op by a gynaecologist using wooden spatulas Two slides are collected per patient. One of slides is immediately wet fixed in $95 \%$ ethanol. It is labeled as wet fixed (WF).

The other slide was air dried for 30 minutes at room temperature and rehydrated for prior to routine staining This one is rehydrated by using glycerol saline rehydration method by 10 dips in $10 \%$ glycerol and wet fixed. Then the slides were coded, and stained by standard Papanicolaou method. The smears are divided into WF and ARF groups. All the paired slides were used for statistical analysis.

The new Bethesda system of reporting cervicovaginal smears (2014) is used for diagnosis, and we assessed different parameters such as cellularity, cytolysis, cell borders, cytoplasmic staining, appearance of chromatin, nuclear borders and presence or absence of red blood cells. Using SPSS software, version 13, a comparison of two major groups can be made using the chi $^{2}$ test. $\mathrm{P}$ value less than 0.005 is considered significant

\section{Results}

We got the following results after comparing the paired slides of WF and ARF pap smears in 300 cervical smears. Approximately half of the patients (51\%) included in the study belonged to 35- 45-years of age years (table -1$)$. Out of 300 females 192 were in the reproductive age group (64\%). Most of them (58\%) came to the outpatient department with complaints of discharge per vaginum or post-menopausal bleeding (19\%) (Table -2). Majority of the patients were diagnosed to have normal or inflammatory smear with very few results of LSIL or HSIL.

Table-1: Age distribution of the patients.

\begin{tabular}{|c|c|c|}
\hline Age & Number & Percentage \\
\hline$<25$ & 4 & 1.3 \\
\hline $25-35$ & 36 & 12 \\
\hline $35-45$ & 152 & 51 \\
\hline $45-55$ & 67 & 22 \\
\hline $55-65$ & 34 & 11.3 \\
\hline$>65$ & 7 & 2.3 \\
\hline
\end{tabular}


Table-2: Chief complaints of the subjects.

Original Research Article

\begin{tabular}{|c|c|c|}
\hline Complaints & Number & Percentage \\
\hline Vaginal discharge & 174 & 58 \\
\hline Menorrhagia & 38 & 12.6 \\
\hline Post coital bleeding & 19 & 6.3 \\
\hline Post menopausal bleeding & 58 & 19.5 \\
\hline Abdominal pain & 11 & 3.6 \\
\hline
\end{tabular}

Comparing the cytomorphology of the cells, cytoplasmic staining found to be better in ARF smears compared to WF smears. Both squamous, glandular cells and inflammatory cells were showed good quality staining. ARF smears were also found to have cells with distinct nuclear borders, clear background and low rate of cytolysis.

Even though previous studies showed higher rates of cytolysis resulting from glycerol rehydration our study results seen to be satisfactory. Clear background resulted from lysis of RBCs during rehydration which made interpretation of smears easier. Factors like cellularity, cell borders and appearance of chromatin showed comparable results in both group and were not statistically significant.

\section{Discussion}

Pap smear is routinely used worldwide for early detection of precancerous lesions and inflammatory conditions of uterus, cervix and vagina. Smears are collected from the female genital tract, fixed stained and screened under a microscope. A Pap smear is a medical procedure in which sample of cells from a woman's cervix is collected by an instrument called Ayers spatula and smeared on a slide. Pap test is the foundation of cervical cancer screening [7]. It is a cheap, simple, painless, screening test which is used to diagnose most of the benign and malignant conditions of the female genital tract and is commonly used to determine intraepithelial cell abnormalities. This screening test is introduced by Dr. George. N. Papanicolaou. He is the father of exfoliative cytology. Introduction of this valuable screening test further led to the remarkable decline in the mortality percentage due to cervical cancer in many developed and developing countries. Same method is used in exfoliative cytology also [8].

The routine is to take the smears under direct vision using Ayres wooden spatula, from the female reproductive tract, smear the slide and then immediately wet fix it in 95\% ethyl alcohol and send them to the laboratory, were they are stained and evaluated by the cytopathologist by pap stain. The stain, which was invented by Papanicolaou bearing his name, was now universally adopted in processing cervicovaginal smears. To detect the neoplasia after hysterectomy vaginal vault smears are taken [9].

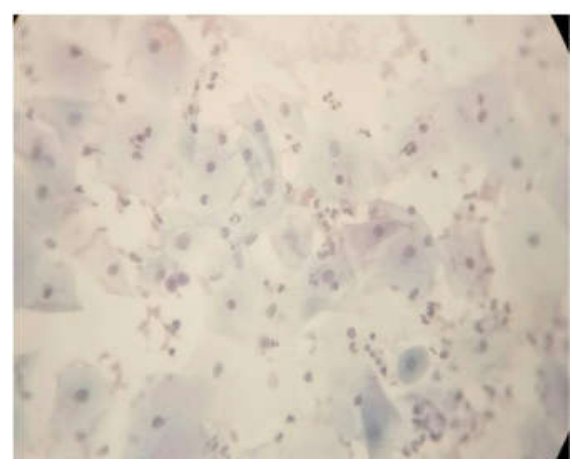

Figure 1. Air dried cervical smears showing squamous cells with better cytoplasmic staining and distinct nuclear borders

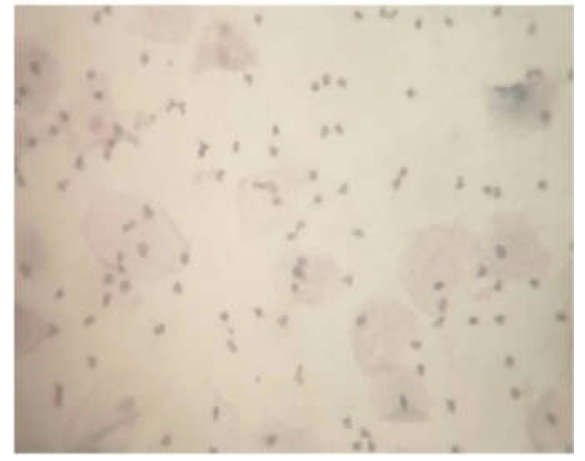

Figure-2: Wet fixed cervical smear showing squamous cells with faint cytoplasmic staining and hazy nuclear borders compared to air dried smears

For years it is known that $\mathrm{H}$ and $\mathrm{E}$ and Pap are the best stains for cytology smears for maintaining cellular resemblance with the same cells in tissue sections [10]. The pap stain is considered to be excellent in demonstrating nuclear morphology and also in maintaining cytoplasmic translucency. But this stain demanded immediate fixation, routinely done in $95 \%$ ethanol. 


\section{Original Research Article}

The conventional Pap stain should never be allowed to dry, starting from collection until cover slipping because it can lead to morphological changes in cells $\mathrm{s}^{\mathbf{8}}$ and air drying artefacts are found to be very common. In cytological examination, especially in aspiration cytology with small amount of aspirate, the material is dry particularly at the edges of the smears, it is highly desirable to develop methods to rehydrate these dried-up diagnostic cells [11]. The need for an alternate method like rehydration of air-dried smears raised in early 1960s. Different studies used various methods like sequential tap-water and acetic acid-alcohol solutions, 50\% aqueous glycerine, hydroxypropyl methylcellulose ether etc. [12,13]. But these methods were not found to be successful may be because of availability of the muchsimpler spray fixatives [14]. The present study was done to know the effectiveness of air driedrehydrated smears in the early diagnosis of insitu and invasive lesion of the cervix. This rehydration methods are very useful in mass population screening programmes in developing countries like India, where there is deficiency of trained staffs, coupling jars and $95 \%$ ethyl alcohol.

In this study we collected both wet fixed and air dried smears and compared after staining cervical smears air dried carefully for two hoursand then rehydrated with saline and 50\% glycerine. There is no time limit set for duration of air drying vary from 30 minutes to one day but longer duration may lead to autolytic changes in these unfixed cells resulting in less satisfactory staining. The rehydration obtained by using glycerol saline rehydration method were slides dipped 10 times in $10 \%$ glycerol followed by wet fixation. Our results showed that cytoplasmic staining was excellent in both squamous and glandular cells. Dipping in aqueous glycerine caused haemolysis so that the background was free of red blood cells, it is seen that minimum of 30 seconds is needed for lysis of the red blood cells and longer immersion can cause nuclear wrinkling, particularly in the lymphoid cells. The clean background and reduced drying artefact resulted in increased the cellular cytoplasmic staining quality.

It is seen that this method of rehydration has many advantages compared to the conventional wet fixation. This technique will result in more thinly and evenly spread smears where the cells are adhered better to the slide and do not fall off as easily on further rehydration. Another advantage is that the air drying in the edges of the smear which is a common problem with wet smears can be avoided. Again because of lysis of most of the red blood cells background will be clear and cells will appear flatter and the depth of focus on the nuclei is much more shallow. Which is an advantage while on taking photomicrographs. The cost effect of preparing dry rehydrated smears is less and wastage is also reduced since the slide used for spreading he smears may also be stained after rehydration.

Rehydration technique can be used effectively in all fine needle aspiration, intraoperative cytology studies [15,16]. The rehydration method was beneficial for urine and blood-stained body cavity fluids [17]. The immunoreactivity pattern with each immunomarker is affected by the method of cytology smear processing. The method of preparation and fixation had variable effects on the immunoreactivity of marker antibodies. The optimum results were achieved with saline-rehydrated, air-dried smears post-fixed in alcoholic formalin for anti-cytokeratin antibody AE1/AE3 [18]. Most of the other markers Except vimentin, showed equal intensity scores with method A or B [19]. Therefore this method of collecting dry smears and rehydrating further can be used routinely or as an alternative in mass cervical cancer screening programmes where the resources are limited because it is very simple, accurate and easier technique which is superior or comparable to the traditional wet smears

\section{Conclusions}

After comparing the paired slides stained after routine wet fixation method and air dried smears after rehydration, better cytoplasmic staining, distinct nuclear borders and clear background prompted us to conclude that the rehydration of air dried smears can be used as an alternative method especially in mass screening programmes in developing countries like India. It is very simple, accurate and easier technique which is superior or comparable tothe traditional wet smears, so it can be recommended to resource limited settings. This method can be used for fine needle aspiration specimens and fluid smears. Slides stained by rehydration techniques are found to retain the antigens, their by making these smears suitable for immunostaining also.

Contribution: Data collection, cytomorphological analysis and manuscript preparation done by first author. The smear collection done by second author.

Acknowledgment: I express my sincere gratitude to Ms Harsha for statistical support and all teaching and nonteaching staff in pathology department for their support.

Scope of the study and future research: Even though a few studies have published worldwide regarding different fixation methods in cytology, studies from India is very few. Our study showed better cytomorphology with glycerol saline rehydration method, which is a new finding. In future more studies are needed to further test the feasibility of this method and to find out still simple methods. 
Findings: Nil; Conflict of Interest: None initiated Permission from IRB: Yes

\section{References}

1. Sternberg, Stephen.S, Stacey E Mills and Darryl Carter. Sternberg's Diagnostic Surgical Pathology. 5th ed. Philadelphia: Lippincott Williams \& Wilkins, 2010 Chapter 52 The Cervix; p 2150

2.Maitra A, Abbas AK. Robbins and Cotran- Pathologic Basis of Disease. 7th ed. New Delhi: Elsevier; 2004.The female genital system;p.1169-70.

3. Bancroft, J.O, A. Stevens. Theory and Practice of Histological Techniques. 2nd ed. Edinburgh: Churchill Livingstone:1982. P. 466.

4. Sivaraman G, Iyengar KR. Rehydrated air-dried Pap smears as an alternative to wet-fixed smears. Acta Cytol. 2002 Jul-Aug;46(4):713-7.

5. Gupta S, Sodhani P, Chachra KL. Rehydration of airdried cervical smears: a feasible alternative to conventional wet fixation. Obstet Gynecol. 2003 Oct; 102 (4): 761-4.

6. John k. c. chan, Ignatius T. Kung. Rehydration of Air-Dried Smears with Normal Saline. Application in Fine-Needle Aspiration Cytologic Examination. A.J. C.P. $1988 ; 89(1): 30-4$

7. Koss, Leopold G, Melamed, Myron R. Koss Diagnostic Cytology and Its Histopathologic Bases. $5^{\text {th }}$ ed. New york: Lippincott Williams and Wilkins; 2006. Vol 1; p.1-20.

8. EARN AA. The clinical use of exfoliative uterovaginal cytology. Can Med Assoc J. 1952 May;66 (5): 465-8.

9. Lampard-SH, Wilson S, Waddell C, Ryan A, Holder $\mathrm{R}$, Kehoe $\mathrm{S}$ et al. Systemic review: Vaginal vault smears after hysterectomy for reasons other than malignancy: a systemic review of the literature. BJOG 2006; 113:1354-1365.

\section{Original Research Article}

10. Lowhagen T, Willems JS: General comments on aspiration biopsy cytology, Compendium on diagnostic cytology. 5thed. Tutorials of Cytology,1983.p506-511.

11. Graham RM. The cytologic diagnosis of cancer. $2^{\text {nd }}$ ed. Philadelphia: WB Saunders: 1964. p 343-344.

12. Lencioni LJ, Stafneri JJ, Cardonnet LJ. Vaginal and urinary sediment smear staining technique without previous fixation, adapted to Papanicolaou's and Shorr's staining methods. J Lab Clin Med 1954; 44:595-599.

13. Bonime RG. Air-dried smear for cytologic studies. Obstet Gynecol. 1966 Jun;27(6):783-90.

14. Bales CE, Durfee GR. Cytological techniques, Diagnostic cytology and its histopathologic basis. 3rd ed. Philadelphia: JB Lippincott; 1979. p 1187-1266.

15. Chan JK, Kung IT. Rehydration of air-dried smears with normal saline.Application in fine-needle aspiration cytologic examination. Am J Clin Pathol. 1988 Jan; 89 (1): 30-4.

16. Shidham VB, Kampalath B, England J. Routine air drying of all smears prepared during fine needle aspiration and intraoperative cytology studies. An opportunity to practice a unified protocol offering the flexibility of choosing a variety of staining methods. Acta Cytol. 2001 Jan-Feb; 45 (1): 60-8. DOI:10.1159/ 000327188

17. Ng WF, Choi FB, Cheung LL, et al. Rehydration of air-dried smears with normal saline. Application in fluid cytology. Acta Cytol. 1994 Jan-Feb;38(1):56-64.

18. Shidham VB, Lindholm PF, Kajdacsy-Balla A, et al. Methods of cytologic smear preparation and fixation. Effect on the immunoreactivity of commonly used anticytokeratin antibody AE1/AE3. Acta Cytol. 2000 Nov-Dec;44(6):1015-22. DOI:10.1159/000328590

19. Shidham VB, Chang CC, Rao RN, et al. Immunostaining of cytology smears: a comparative study to identify the most suitable method of smear preparation and fixation with reference to commonly used immunomarkers. Diagn Cytopathol. 2003 Oct; 29 (4): 217-21. DOI:10.1002/dc. 10364

\section{How to cite this article?}

Narayanan O. N, Bai A. Comparison of different methods of fixation in Papanicolaou staining of cervical smears: Wetfixation and rehydration of air dried smears. Trop J Path Micro 2019;5(1):15-19.doi:10.17511/jopm.2019.i01.03. 